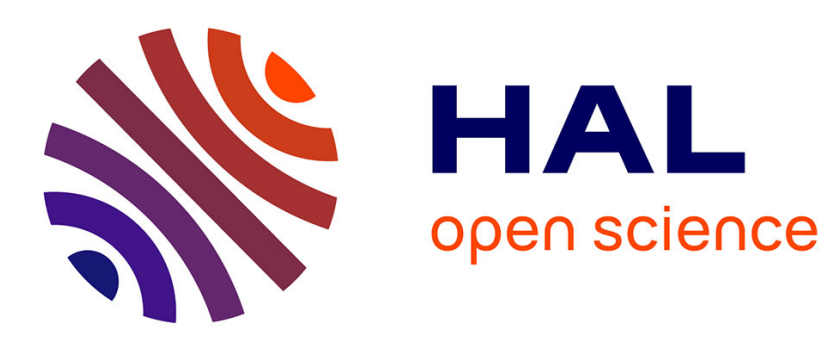

\title{
Fault diagnosis in nonlinear systems through an adaptive filter under a convex set representation
}

Manuel Adam Medina, Mickael Rodrigues, Didier Theilliol, Hicham Jamouli

\section{To cite this version:}

Manuel Adam Medina, Mickael Rodrigues, Didier Theilliol, Hicham Jamouli. Fault diagnosis in nonlinear systems through an adaptive filter under a convex set representation. European Control Conference, ECC 2003, Sep 2003, Cambridge, United Kingdom. pp.CDROM. hal-00364807

\section{HAL Id: hal-00364807 https://hal.science/hal-00364807}

Submitted on 27 Feb 2009

HAL is a multi-disciplinary open access archive for the deposit and dissemination of scientific research documents, whether they are published or not. The documents may come from teaching and research institutions in France or abroad, or from public or private research centers.
L'archive ouverte pluridisciplinaire HAL, est destinée au dépôt et à la diffusion de documents scientifiques de niveau recherche, publiés ou non, émanant des établissements d'enseignement et de recherche français ou étrangers, des laboratoires publics ou privés. 


\title{
FAULT DIAGNOSIS IN NONLINEAR SYSTEMS THROUGH AN ADAPTIVE FILTER UNDER A CONVEX SET REPRESENTATION
}

\author{
M. Adam-Medina, M. Rodrigues, D. Theilliol and H. Jamouli \\ Centre de Recherche en Automatique de Nancy \\ CNRS UMR 7039 \\ B.P. 239, 54506, Vandoeuvre Cedex, France. \\ Phone: 33383684462 - Fax: 33383684461 \\ email: medina@cran.uhp-nancy.fr
}

Keywords: Nonlinear system, multiple linear models, fault detection and isolation, stability, adaptive filter.

\begin{abstract}
In this paper, the main goal is to design an approach that performs fault detection, isolation and estimation for a large class of nonlinear systems. Fault diagnosis is established by regarding system as a convex combination of linear time invariant (LTI) stochastic models and not as a single global model. The nonlinear representation is based on a bank of decoupled Kalman filters. This paper consists in generating a robust model selection of the "best" representative linear model. Under fault isolation conditions, the main contribution is to design an adaptive filter which makes possible multiple faults detection which appear simultaneously or in a sequential way, isolation and estimation over the whole operating range of nonlinear system. The stability conditions of the adaptive filter are developed. These conditions result in convex linear matrix inequalities (LMIs) that can be solved efficiently with optimization techniques. Performances of the method are tested on an academic example.Copyright@2003 EJC.
\end{abstract}

\section{Introduction}

Fault detection and isolation (FDI) has been developed traditionally with model-based approaches using linear or linearized models. Several papers have been published: these works lead to robust methods of parametric uncertainties and system modelling errors when noise is or is not present (CHEN and PATTON, 1999). These diagnosis methods are based on residual generation. One solution is to use nonlinear methods. Fault detection and isolation (FDI) problems for nonlinear systems provide a challenging current research topic (FRANK et al., 2000). (CHEN and PATton, 1999) have proposed FDI scheme using linear observers and Takagi-Sugeno configuration for nonlinear system representation in the deterministic case. This technique is based on the decomposition of the original system into a set of linear models. This structure is considered as multiple model (MM) approach. In nonlinear systems, the MM structure has been proposed for control do- main. The robust control using one controller has been presented in (BANERJEE et al., 1995), the multi-control technique such as gain scheduling interpolation method in control domain (LEITH and LEITHEAD, 2000) and adaptive or learning control based on neural network have been developed (NARENDRA et al., 1995). However, these methods have not been frequently used for FDI. Under this consideration, the paper aims and contributes to the design of a multiple fault detection, isolation adaptive filter in nonlinear system represented as a convex set of LTI distribution matrices. In our approach, the term "adaptive" should not be associated by readers to an on-line parameter estimation as developed by (NARENDRA et al., 1995).

Our goal is to design a scheme which allows FDI and a robust model selection in the nonlinear system represented as a convex combination of the multiple LTI model. To achieve this purpose, an adaptive filter based on an interpolated multiple model is developed. The proposed filter will perform an efficient FDI according to an available convex set representation in faulty case based on decoupled Kalman filter developed by (KELLER, 1999). Moreover, the magnitude, the time of occurrence and the localization of the faults are considered as unknown.

The paper is organised as follows: in the second section, the general problem of the robust selection in nonlinear systems described by MM is developed. A solution based on the design of a bank of decoupled Kalman filters is then developed and justified. This section gives us a formulation of the nonlinear system representation in faulty case based in a convex set of the multiple LTI models. In section III, the design of the adaptive filter is developed. The question of stability was addressed in the terms of Lyapunov quadratic stability by using a LMI technique in Section IV. In next Section, an academic example is considered to illustrate the theoretical results. Finally, the last section is devoted to the conclusions and future search.

\section{Fault Diagnosis in nonlinear systems}

\subsection{Problem statement}

Dynamic systems such as nonlinear, linear time-invariant, linear time-variant, linear piecewise and hybrid can be represented by a decomposition of the full operating range 
into a number of possibly overlapping operating regimes (LEITH and LEITHEAD, 2000). For each regime, a simple local linear system is defined. Let us assume the following general faulty stochastic system as considered by (VARGA, 1996) and (MURRAY-SMITH and JOHANSEN, 1997)

$$
\left\{\begin{aligned}
X_{k+1} & =A_{j} X_{k}+B_{j} U_{k}+\Delta_{X_{j}}+F_{j} d_{k}+\omega_{k}^{j} \\
Y_{k} & =C X_{k}+\Delta_{Y_{j}}+\nu_{k}^{j}
\end{aligned}\right.
$$

where $X \in \Re^{n}$ represents the state vector, $U \in \Re^{p}$ is the input vector, $Y \in \Re^{m}$ is the measurement vector and $d \in \Re^{q}$ is the fault vector. Each linear model is defined around $j^{\text {th }}$ operating point, noted $P_{j} \forall j=[1,2, \cdots, M]$, where $M$ is the total number of operating points. Each operating point is defined by a pair of input/output signals $\left(Y_{P j}, U_{P j}\right)$. The terms $\omega^{j}$ and $\nu^{j}$ are two independent zero mean white noises with variance-covariance matrices defined respectively by $Q^{j}$ and $R^{j} . A_{j}, B_{j}$, and $C$ are constant matrices with appropriate dimensions. Around the $j^{\text {th }}$ operating point it is assumed that $\forall j, \operatorname{rank}(C)=m . \Delta_{X_{j}}$ and $\Delta_{Y_{j}}$ are contant vectors depending on the $j^{\text {th }}$ linear model. $F_{j}$ is the fault distribution matrix representing actuator, sensor or component faults with $\forall j, \operatorname{rank}\left(F_{j}\right)=q$. Without loss of generality according to (PARK et al., 1994), in the presence of sensor, actuator or component faults, the system may be represented by a linear system as (1). This linear system (1) can be specified by the set of system matrices as:

$$
S_{j}=\left[\begin{array}{ccccc}
A_{j} & B_{j} & \Delta_{X_{j}} & F_{j} & \omega^{j} \\
C & \Delta_{Y_{j}} & \nu^{j} & &
\end{array}\right], \forall j=[1,2, \ldots, M]
$$

Let $S_{k}$ be a matrix sequence varying within a convex set, defined as:

$$
S_{k}:=\left\{\sum_{j=1}^{M} \varphi_{k}^{j} S_{j}: \varphi_{k}^{j} \geq 0, \sum_{j=1}^{M} \varphi_{k}^{j}=1\right\}
$$

In the MM framework, $S_{k}$ characterizes at each sample the nonlinear system. Consequently, the dynamic behavior of nonlinear system can be defined by a convex set of a multiple LTI models, noted $\Upsilon\left(\Upsilon:=\left\{\left[S_{1}, S_{2}, \ldots S_{M}\right]\right\}\right)$.

The state space representation (3) under a convex set can be considered as:

- a conventional modelling approach for non linear smooth plant where $\varphi_{k}^{j}$ is an appropriate weighting functions. The functions $\varphi_{k}^{j}$, which can be a vector or a scalar, embodies the nonlinearity of the plant as discussed in fault free case (LEITH and LEITHEAD, 2000) or (MURRAY-SMITH and JOHANSEN, 1997).

In order to achieve multi-control and FDI, a bank of Kalman filter is designed. Under assumption that the nonlinear system evolves around the $j^{\text {th }}$ operating point, the Kalman filter is described by:

$$
\left\{\begin{aligned}
\hat{X}_{k+1}^{i} & =A_{i} \hat{X}_{k}^{i}+B_{i} U_{k}+\Delta_{X_{i}}+K_{k}^{i}\left(Y_{k}-\hat{Y}_{k}^{i}\right) \\
\hat{Y}_{k}^{i} & =C \hat{X}_{k}^{i}+\Delta_{Y_{i}}
\end{aligned}\right.
$$

where $\hat{X}_{k}^{i} \in \Re^{n}$ denotes the estimated state vector and $\hat{Y}_{k}^{i} \in \Re^{m}$ is the output estimation obtained from the linear filter based on the $i^{\text {th }}$ linear model. $K_{k}^{i} \in \Re^{n \times m}$ is the Kalman filter gain matrix. In this paper, the fault distribution matrix $F_{j}$ is also is represented as $F_{i}$ with the same rank.

In the MM framework, the construction of a bank of Kalman filters defined from $\Upsilon, \forall i=[1,2, \ldots, M]$ is considered in order to establish fault detection, isolation and the robust model selection on all the operating range of the system. Based in the needs to develop a robust model selector in faulty or free case, a bank of Kalman filters leads us to obtain the estimated error $\left(\varepsilon_{k}^{i} \quad\left(\varepsilon_{k}^{i}=X_{k}-\hat{X}_{k}^{i}\right)\right.$ and the output residual vectors $\left.r_{k}^{i} \quad\left(r_{k}^{i}=Y_{k}-\hat{Y}_{k}^{i}\right)\right)$ when the both fault and operating point change occurs $(j \neq i$ and $d \neq 0$ ). This difference between the representation system to be considered and the filters is represented by equation (5) and (6) as:

$$
\begin{gathered}
\varepsilon_{k+1}^{i}=\left(A_{i}-K_{k}^{i} C\right) \varepsilon_{k}^{i}+F_{j} d_{k} \\
-K_{k}^{i} \nu_{k}^{j}+\omega_{k}^{j}+\left(\Delta \Delta_{X_{j}}^{i}-K_{k}^{i} \Delta \Delta_{Y_{j}}^{i}\right) \xi_{j, k}^{i}
\end{gathered}
$$

and,

$$
r_{k}^{i}=C_{i} \varepsilon_{k}^{i}+\nu_{k}^{j}+\Delta \Delta_{Y_{j}}^{i} \xi_{j, k}^{i}
$$

where $\xi_{j, k}^{i} \in \Re^{(n+p+1) \times 1}$ corresponds to the uncertainties magnitude between nonlinear system represented by the $j^{\text {th }}$ linear system and the $i^{\text {th }}$ linear model used for the Kalman filters computation (i.e., operating point switches). $\Delta \Delta_{X_{j}}^{i} \in \Re^{n \times(n+p+1)}$ and $\Delta \Delta_{Y_{j}}^{i} \in \Re^{m \times(n+p+1)}$ are the distribution matrices of uncertainties associated with the state system equation and the output equation, respectively. Equations (5) and (6) show that residual is sensitive to modelling errors (i.e, a change in the operating point) and at the same time to faults. The use of Kalman filters leads to the following residual properties $(\forall i \in[1, \cdots, M])$ :

$$
\begin{array}{ll}
r_{k}^{i} \sim \mathcal{N}, & \text { if } d=0, i=j \\
r_{k}^{i} \nsim \mathcal{N}, & \text { if } d=0, i \neq j \\
r_{k}^{i} \nsim \mathcal{N}, & \text { if } d \neq 0, \forall i
\end{array}
$$

In faulty case $(d \neq 0)$ and whatever the operating point, direct application of nonlinear system using Kalman filters based on a MM framework can be considered, but at the cost of certain degradations in performance. Indeed, in faulty cases, FDI cannot be done correctly since the residual vector is corrupted by two pieces of information at the same time: operating point exchanges and fault occurrences. The probabilistic Bayes method cannot define the best representative model (ADAM-MEDINA et al., 2003). Firstly, in this paper, we are interested in knowing which linear model is more representative at each time both in fault free and faulty cases. These considerations yield a new residual generator definition allowing the decoupling of fault effects and modelling errors. This new residual generator gives a first signal insensitive to faults, but sensitive to modelling errors and a second signal sensitive to faults. The new residual generator is expressed as:

$$
\tilde{r}_{k}^{i}=\left[\begin{array}{l}
\Sigma \\
\Xi
\end{array}\right] r_{k}^{i}
$$

where $\Sigma$ and $\Xi$ are terms introduced in order to decouple the residuals with appropriate dimensions and $\tilde{r}_{k}^{i}$ is the new 
residual vector. One solution is proposed in the following paragraph.

\subsection{Robust model selection in free and faulty case.}

Under the hypotheses that a fault occurs at time $k_{d}(k>$ $\left.k_{d}\right)$, and that operating point switches at time $k_{e}\left(k>k_{e}\right)$, the residual vector of the $i^{\text {th }}$ filter is expressed as:

$$
\begin{aligned}
& r_{k}^{i}=\bar{r}_{k}^{i}+\Delta \Delta^{i} \xi_{j, k}^{i} \\
& \quad+\quad \rho_{k, k_{d}}\left[d_{k_{d}} d_{k_{d}+1} \cdots d_{k-1}\right] \\
& \quad+\quad \beta_{k, k_{e}}\left[\xi_{j, k_{e}}^{i} \xi_{j, k_{e}+1}^{i} \cdots \xi_{j, k-1}^{i}\right]
\end{aligned}
$$

where $\rho_{k, k_{d}}, \beta_{k, k_{e}}, \Gamma_{i, k}^{\left(k_{d}, k_{e}\right)}, L_{k}^{i}$ are matrices with appropriate dimensions. Equation (9) allows us to confirm that residual is affected by both fault effects and modelling errors. We wish to generate residuals insensitive to fault effects but sensitive only to modelling errors, that is:

$$
\left(A_{i}-K_{k}^{i} C\right) F_{i}=0
$$

Supposing that condition (10) is fulfilled and the number of fault is strictly lower than the number of outputs (i.e., $\forall i, \operatorname{rank}\left(C F_{i}\right)=q<m$ ), a solution was proposed by (KELLER, 1999) which defined a Kalman filter gain as:

$$
K_{k}^{i}=\omega_{i} \Xi_{i}+\bar{K}_{k}^{i} \Sigma_{i}
$$

with $\Xi_{i}=\left(C F_{i}\right)^{+}, \omega_{i}=A_{i} F_{i}, \Sigma_{i}=\alpha_{i}\left(I_{m}-C F_{i} \Xi_{i}\right)$ and $\alpha_{i} \in \Re^{(m-q) \times m}$ is an arbitrary constant matrix defined so that the matrix $\Sigma_{i}$ is of full row rank. According to (11) and the previous matrices properties, the residual vector $\tilde{r}_{k}^{i}$ can be obtained as suggested in (8):

$$
\left[\begin{array}{c}
\Sigma_{i}\left(Y_{k}-\hat{Y}_{k}^{i}\right) \\
\Xi_{i}\left(Y(k)-\hat{Y}_{k}^{i}\right)
\end{array}\right]=\left[\begin{array}{c}
\Sigma_{i} r_{k}^{i} \\
\Xi r_{k}^{i}
\end{array}\right]=\left[\begin{array}{c}
\gamma_{k}^{i} \\
\Omega_{k}^{i}
\end{array}\right]=\tilde{r}_{k}^{i}
$$

where $\gamma_{k}^{i} \in \Re^{m-q}$ is the residual vector decoupled to fault and $\Omega_{k}^{i} \in \Re^{q}$ is the residual vector sensitive to fault. Following these assumptions, when nonlinear system operates around the $j^{\text {th }}$ operating point, the new residual $\gamma_{k}^{i}$ insensitive to fault has the following properties:

$$
\forall d\left\{\begin{array}{l}
\gamma_{k}^{i} \sim \mathcal{N} \text { if } i=j \\
\gamma_{k}^{i} \nsim \mathcal{N} \text { if } i \neq j
\end{array}\right.
$$

where $\Theta_{k}^{i}$ defines the covariance matrix of the residuals $\gamma_{k}^{i}$, equal to $\left(\bar{C} \bar{P}_{k}^{i} \bar{C}^{T}+\bar{V}_{i}\right)$. Considering that residual $\gamma_{k}^{i}$ around $j^{\text {th }}$ operating point follows a Gaussian distribution, in the same way as (Banerjee et al., 1995) the residuals vector can then be used to compute the probability distribution as:

$$
\wp_{k}^{i}=\frac{\exp \left\{-0.5 \gamma_{k}^{i}\left(\Theta_{k}^{i}\right)^{-1}\left(\gamma_{k}^{i}\right)^{T}\right\}}{\left[(2 \pi) \operatorname{det}\left(\Theta_{k}^{i}\right)\right]^{1 / 2}}
$$

The mode probability decoupled from faults is expressed as:

$$
\varphi\left(\gamma_{k+1}^{i}\right)=\frac{\wp_{k}^{i} \varphi\left(\gamma_{k}^{i}\right)}{\sum_{l=1}^{M} \wp_{k}^{l} \varphi\left(\gamma_{k}^{l}\right)}
$$

The probability algorithm allows to obtain the $i^{\text {th }}$ linear model describing the system behavior in both fault free and faulty cases. The mode probabilities allows us to determine the operating point where nonlinear system is evolving. These probabilities are used to isolate the operating point and consequently define a robust model selector.

The robust model selector is used to represent the plant behavior as a convex set of multiple linear models. This representation is described in equation (16):

$$
S_{k}^{\star}:=\left\{\sum_{i=1}^{M} \varphi\left(\gamma_{k}^{i}\right) S_{i}: \varphi\left(\gamma_{k}^{i}\right) \geq 0, \sum_{i=1}^{M} \varphi\left(\gamma_{k}^{i}\right)=1\right\}
$$

where $S^{\star}(k)$ represents the global model and $S_{i}$ is defined as:

$$
S_{i}=\left[\begin{array}{cccc}
A_{i} & B_{i} & \Delta_{X_{i}} & F_{i} \\
C & \Delta_{Y_{i}} & &
\end{array}\right], \forall i=[1,2, \ldots, M]
$$

By taking in to account the nonlinear system convex set notation in (16), the nonlinear state space representation is then defined as:

$$
\left\{\begin{aligned}
X_{k+1} & =A_{k}^{\star} X_{k}+B_{k}^{\star} U_{k}+\Delta_{X, k}^{\star}+F_{k}^{\star} d_{k} \\
Y_{k} & =C X_{k}+\Delta_{Y, k}^{\star}
\end{aligned}\right.
$$

$$
\begin{aligned}
\text { with } & =\sum_{i=1}^{M} \varphi\left(\gamma_{k}^{i}\right) A_{i}, & B_{k}^{\star}=\sum_{i=1}^{M} \varphi\left(\gamma_{k}^{i}\right) B_{i} \\
\Delta_{X, k}^{\star} & =\sum_{i=1}^{M} \varphi\left(\gamma_{k}^{i}\right) \Delta X_{i}, & \Delta_{Y, k}^{\star}=\sum_{i=1}^{M} \varphi\left(\gamma_{k}^{i}\right) \Delta Y_{i} \\
F_{k}^{\star} & =\sum_{i=1}^{M} \varphi\left(\gamma_{k}^{i}\right) F_{i} &
\end{aligned}
$$

The equations (18) and (19) represent the original nonlinear system. In this representation, the noisy function has already been considered in the convex set representation. This convex set representation is used for the design of an adaptive filter which is developed for the fault detection, isolation and estimation (see Fig.1) in the following section.

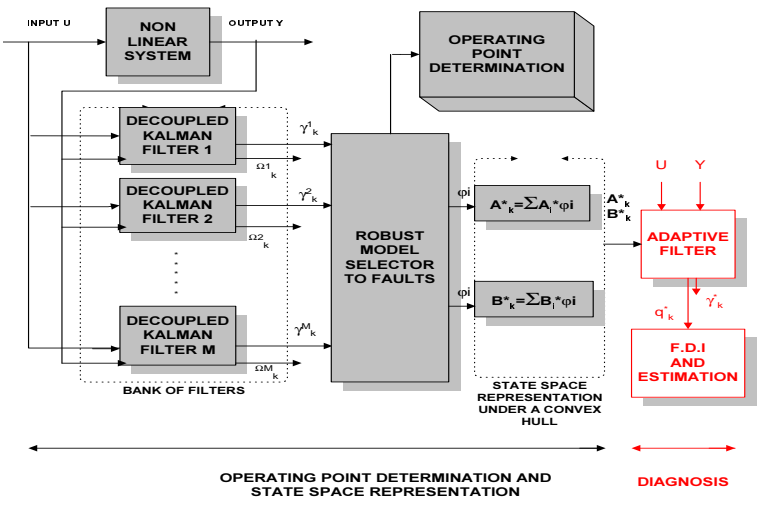

Figure 1: FDI and nonlinear representation scheme 


\section{Adaptive filter}

\subsection{A new convex representation of the nonlinear sys- tem}

To design the adaptive filter, an unique formulation of the convex faulty representation is proposed. In the state space representation (18), a matrix $F_{k}^{\star}$ is calculated as: $F_{k}^{\star}=\sum_{i=1}^{M} \varphi\left(\gamma_{k}^{i}\right) F_{i}$ where matrix $F_{i} \in \mathbb{R}^{n \times q}$ is fault distribution matrix of each model $i$. Faults contribution on the system is described into state space representation as: $\left(\sum_{i=1}^{M} \varphi\left(\gamma_{k}^{i}\right) F_{i}\right) d_{k}$.

According to some $F_{i}$ matrix conditions, the convex combination can be transformed into an unique form:

Definition 1 Matrix $F_{i}^{h}$ (respectively $\Im^{h}$ ) defines the $h^{\text {th }}$ column of matrix $F_{i}$ (respectively $\Im$ ).

Theorem $1 \forall h \in[1 . . q], \forall i \in[1 . . M]$, with $\operatorname{Rank}\left[F_{i}\right]=q$ if $\operatorname{Rank}\left[F_{1}^{h} . . F_{i}^{h} . . F_{M}^{h}\right]=1$, then

$\left(\sum_{i=1}^{M} \varphi\left(\gamma_{k}^{i}\right) F_{i}\right) d_{k}=\Im f_{k}$

where $d \in \mathbb{R}^{q}$ represents "true" fault vector and $f \in \mathbb{R}^{q}$ is an image of fault vector, where $\Im \in \mathbb{R}^{n \times q}$ is a constant fault distribution matrix which columns vectors get direction of columns vectors of matrices $F_{i}$.

\section{Proof (Theilliol et al., 2003)}

\subsection{Adaptive filter design}

In order to detect and isolate faults, a classical discret filter with a gain $K_{k}$ could be designed according to matrices $A_{k}^{\star}$ and $C$ defined in (18) and (19):

$\left\{\begin{aligned} \hat{X}_{k+1} & =A_{k}^{\star} \hat{X}_{k}+B_{k}^{\star} U_{k}+\Delta_{X, k}^{\star}+K_{k}\left(Y_{k}-\hat{Y}_{k}\right) \\ \hat{Y}_{k} & =C \hat{X}_{k}+\Delta_{Y, k}^{\star}\end{aligned}\right.$

where $\hat{X}$ and $\hat{Y}$ represent the estimated state and the estimated output.

According to (20) estimation error $e_{k}\left(e_{k}=X_{k}-\hat{X}_{k}\right)$ and output residual $r_{k}\left(r_{k}=Y_{k}-\hat{Y}_{k}\right)$ are expressed as:

$$
\left\{\begin{array}{l}
e_{k+1}=\left(A_{k}^{\star}-K_{k} C\right) e_{k}+\Im f_{k} \\
r_{k}=C e_{k}
\end{array}\right.
$$

Under the assumption that a fault occurs at time $k_{d}(k>$ $k_{d}$ ), residual vector is defined as:

$$
r_{k}=\bar{r}_{k}+\rho_{k, k_{d}}\left[f_{k_{d}} f_{k_{d}+1} \cdots f_{k-1}\right]
$$

where $\bar{r}_{k}$ represents the residual in fault free case and

$$
\rho_{k, k_{d}}=C\left[\begin{array}{c}
\Gamma_{k}^{k_{d}+1} \Im \\
\Gamma_{k}^{k_{d}+2} \Im \\
\ldots \\
\Im
\end{array}\right]
$$

with

$$
\begin{aligned}
\Gamma_{k}^{k_{d}} & =\prod_{\tau=k_{d}}^{k-1} L_{\tau} \\
L_{k} & =\left(A_{k}^{\star}-K_{k} C\right)
\end{aligned}
$$

As developed by (KELLER, 1999), we proposed to design the gain $K_{k}$ such that $\left(\left(A_{k}^{\star}-K_{k} C\right) \Im\right)$ is equal to zero. Under the general classical condition, that the number of faults must not be greater than the number of measurements (i.e. $\operatorname{rank}(C \Im)<m$ ), a fault diagnosis adaptive filter is designed with the following gain definition:

$$
K_{k}=\omega_{k} \Pi+\bar{K}_{k} \Sigma
$$

with $\Pi=(C \Im)^{+}, \omega_{k}=A_{k}^{\star}$, and $\Sigma=\alpha\left(I_{m}-C \Im \Pi\right)$ where $\alpha$ is an arbitrary matrix determined so that matrix $\Sigma$ is of full row rank.

According to (26), the adaptive filter is defined as:

$$
\left\{\begin{aligned}
\tilde{X}_{k+1} & =A_{k}^{\star} \widetilde{X}_{k}+B_{k}^{\star} U_{k}+\Delta_{X_{, k}}^{\star} \\
& +\left(\omega_{k} \Pi+\bar{K}_{k} \Sigma\right)\left(Y_{k}-\widetilde{Y}_{k}\right) \\
\tilde{Y}_{k} & =C \widetilde{X}_{k}+\Delta_{Y, k}^{\star}
\end{aligned}\right.
$$

where $\tilde{X}_{k}$ and $\widetilde{Y}_{k}$ are respectively the estimated state and the estimated output.

The gain decomposition, defined in (26), involves the following matrices properties

$$
\Pi C \Im=I \text { and } \Sigma C \Im=0
$$

and makes possible the generation of modified residual vector:

$$
\left[\begin{array}{c}
\gamma_{k}^{\star} \\
q_{k}^{\star}
\end{array}\right]=\left[\begin{array}{c}
\Sigma \\
\Pi
\end{array}\right] r_{k}=\left[\begin{array}{c}
\Sigma \bar{r}_{k} \\
\Pi \bar{r}_{k}+f_{k-1}
\end{array}\right]
$$

It must be noticed that $\gamma_{k}^{\star} \in \mathbb{R}^{m-q}$ is a residual vector insensitive to faults and $q_{k}^{\star} \in \mathbb{R}^{q}$ is a residual vector sensitive to faults and defines also an fault estimation vector.

The gain $\bar{K}_{k}$, in (26), is the unique degree of freedom in the adaptive filter synthesis. In the multiple model framework, it is designed such as an interpolation of gain $\bar{K}_{i}$ (see (STIWELL and RUGH, 1999) or (LEITH and LEITHEAD, 2000)). Then, in the following, $\bar{K}_{k}$ is noted $\bar{K}_{k}^{\star}$. For the determination of $\bar{K}_{k}^{\star}$, each $\bar{K}_{i}$ is computed off-line by a classical eigenstructure assignment of $\left(\bar{A}_{i}-\bar{K}_{i} \bar{C}\right)$ with $\bar{C}=C \Im$ and $\bar{A}_{i}=A_{i}(I-\Im \Pi C)$. We are now expressed necessary and sufficient conditions for adaptive filter stability.

\section{Stability on a convex hull}

Using Lyapunov stability definition, the gain $\bar{K}_{i}$, establishing off-line by eigenstructure assignement in order that matrix $\left(\bar{A}_{i}-\bar{K}_{i} \bar{C}\right)$ is Hurtwitz, must verified and solved the following inequality :

$$
\left\{\begin{array}{c}
\left(\bar{A}_{i}-\bar{K}_{i} \bar{C}\right)^{T} P\left(\bar{A}_{i}-\bar{K}_{i} \bar{C}\right)-P<0 \\
P>0
\end{array}\right.
$$


A Schur complement operation transforms the inequality (30) into an LMI and as proposed for LPV system in fault free case by (DAAFOUZ and BERNUSSOU, 2001) or (OLIVEIRA et al., 1999), this inequality can be extended on convex sets $\Gamma$ (idem for $\bar{K}_{k}^{\star}$ ) and a new LMI is generated:

$$
\left(\begin{array}{cc}
P & \sum_{i=1}^{M} \varphi\left(\gamma_{k}^{i}\right)\left(\bar{A}_{i}-\bar{K}_{i} \bar{C}\right)^{T} P \\
P \sum_{i=1}^{M} \varphi\left(\gamma_{k}^{i}\right)\left(\bar{A}_{i}-\bar{K}_{i} \bar{C}\right) & P
\end{array}\right)>0
$$

$$
\Gamma=\left\{\bar{A}_{k}^{\star}: \bar{A}_{k}^{\star}=\sum_{i=1}^{M} \varphi\left(\gamma_{k}^{i}\right) \bar{A}_{i}, \sum_{i=1}^{M} \varphi\left(\gamma_{k}^{i}\right)=1\right\}
$$

Eq.(31) is equivalent to:

$$
\left(\begin{array}{cc}
P & \left(\bar{A}_{k}^{\star}-\bar{K}_{k}^{\star} \bar{C}\right)^{T} P \\
P\left(\bar{A}_{k}^{\star}-\bar{K}_{k}^{\star} \bar{C}\right) & P
\end{array}\right)>0
$$

Then, (33) is equivalent to Lyapunov quadratic stability in discrete case of matrice $\left(A_{k}^{\star}-K_{k}^{\star} C\right)$ :

$$
\left\{\begin{array}{c}
\left(A_{k}^{\star}-K_{k}^{\star} C\right)^{T} P\left(A_{k}^{\star}-K_{k}^{\star} C\right)-P<0 \\
P>0
\end{array}\right.
$$

Thus, find an unique solution $P$ for all $[i=1 \ldots M]$ assures the stability of the filter (27) and the convergence of error estimation defined as follows:

$$
\bar{e}_{k+1}=\left(A_{k}^{\star}-K_{k}^{\star} C\right) \bar{e}_{k}
$$

The stability of the adaptive filter has been analyzed and correspond to find an unique matrix to solve a set of inequality. To show the performances of this method an academic example is illustrated in the next section.

\section{Example}

\subsection{System representation}

The proposed method is applied to an open loop discrete nonlinear system. This stochastic discret system, SIMO (single-input/multiple-output) with $X \in \Re^{4}, U \in \Re^{1}$, and $Y \in \Re^{3}$. The $j^{\text {th }}$ linear system represents the system behavior around the $j^{\text {th }}$ operating point depending directly on the magnitudes of the inputs defined in table 1. Other ma-

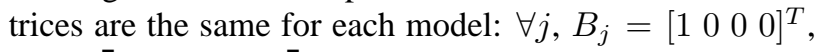
$C=\left[\begin{array}{llll}0 & 1 & 0 & 0 \\ 0 & 0 & 1 & 0 \\ 0 & 0 & 0 & 1\end{array}\right], Q_{j}=\operatorname{diag}\left(\left[\begin{array}{llll}0.9 & 0 & 0.5 & 0.3\end{array}\right]\right)^{T}$ and

\begin{tabular}{|c|c|c|c|}
\hline $\begin{array}{l}\text { Operating } \\
\text { point }\left(P_{j}\right)\end{array}$ & $\begin{array}{c}j=1 \\
U \in[0 ; 3[\end{array}$ & $\begin{array}{c}j=2 \\
U \in[3 ; 6[\end{array}$ & $\begin{array}{c}j=3 \\
U \in[6 ; 9[\end{array}$ \\
\hline$Y_{P_{j}}$ & $\begin{array}{c}3.2-5.0 \\
2.58\end{array}$ & $\begin{array}{c}5.82-8.54 \\
4.88\end{array}$ & $\begin{array}{c}9.88-15.62 \\
8.34\end{array}$ \\
\hline$U_{P_{j}}$ & 2 & 4 & 7 \\
\hline $\begin{array}{c}\text { Eigenvalues } \\
\text { of } A_{j}\end{array}$ & $\begin{array}{l}0.15 \\
0.37 \\
0.60 \\
0.22\end{array}$ & $\begin{array}{l}0.01 \\
0.02 \\
0.04 \\
0.01\end{array}$ & $\begin{array}{l}0.20 \\
0.50 \\
0.80 \\
0.30\end{array}$ \\
\hline
\end{tabular}
$R_{j}=I_{3 \times 3}$.

The faulty distribution matrices are different:

$$
F_{1}=\left[\begin{array}{cc}
0 & 0 \\
0.9 & 0 \\
0 & 2.8 \\
0 & 0
\end{array}\right], F_{2}=\left[\begin{array}{cc}
0 & 0 \\
2.4 & 0 \\
0 & 1.4 \\
0 & 0
\end{array}\right] \text { and } F_{3}=\left[\begin{array}{cc}
0 & 0 \\
3.2 & 0 \\
0 & 3.7 \\
0 & 0
\end{array}\right]
$$

Table 1: Definition of the operating points.

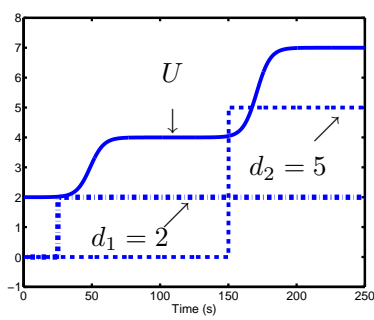

(a) System input $U$ and fault occurrences

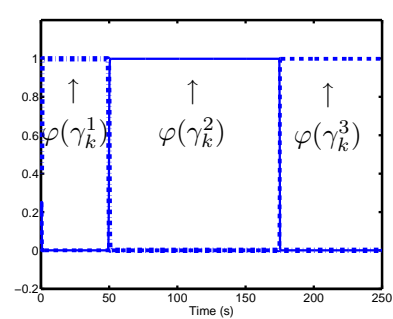

(b) Probabilities $\varphi\left(\gamma_{k}^{i}\right)$
Figure 2: System input and model selector evolution in faulty case.

\subsection{Simulation results}

The simulation example considered is developed in a multiple faults case $\left(d \in \Re^{2}\right)$ with a system input $U$ chosen as successive steps with smooth transitions (see Fig. 2a). In order to evaluate the method, we consider two faults : a first at time $k_{d}=25$ seconds with a constant magnitude equal to 2 , and a second appears at time $k_{d}=150$ seconds with a constant magnitude equal to 5 as shown on Fig. 2a.

A bank of three decoupled Kalman filters in multiple models framework is proposed. It was developed to show the selection of the true model and nonlinear representation, when residual vector is corrupted by two pieces of information: operating point evolution and fault occurrence. In Fig. 2b, we can observe that in the faulty case, how the probabilities evolution allow the robust model selection. The results obtained show a robust model selector which is insensitive to faults. The same figure shows the transition from model " 1 " to model "2" and after to model "3" without the probabilities being affected by fault occurrences. The model selection allows to represent the nonlinear system via a convex set of the multiple LTI models. This representation is used to realize the fault detection, isolation and estimation via an adaptive filter. In FDI context and according to adaptive filter design, different conditions are verified: $\operatorname{rank}(C \Im)<3$, Theorem 1 is available, $\forall i,\left(\bar{A}_{i}, \bar{C}\right)$ is observable and three gains $\bar{K}_{i}$ are computed.

As illustrated in Fig. 3a and after a pseudo-inverse matrix computation (THEILliol et al., 2003) that permits the obtention of accurate fault magnitude estimation where $d$ 


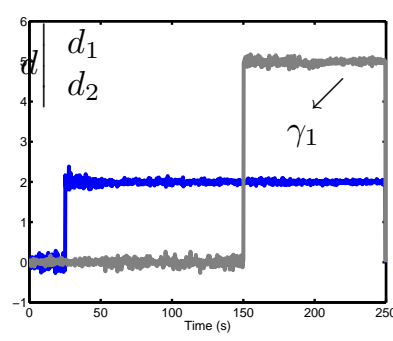

(a) Faults estimation

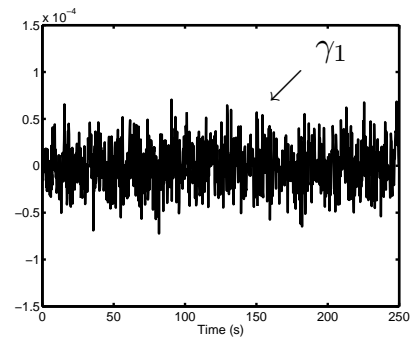

(b) Insensible residual generated by adaptive
Figure 3: Residuals generated by adaptive filter

is a fault estimation of $q_{k}^{\star}$. The good performances of the adaptive filter in terms of fault detection, fault isolation is achieved. Based on this residual vector, it is easy to imagine a residual evaluation test with an elementary logic decision in order to develop a multiple faults detection, isolation and estimation module integrated in supervision platform.

Fig. 3b illustrates the residual vector $\gamma_{k}^{\star} \in \Re^{1}$. In this academic example, where uncertainties or error models are not considered, these residuals vector, designed as a sequence insensitive to faults, are obviously close to zero during the experiment. Meanwhile, in practical case, the residual vector $\gamma_{k}^{\star}$ will not be close to zero due to uncertainties or model errors but it can be exploited to indicate the confidence degree of the adaptive filter.

\section{Conclusion}

The FDI problem for nonlinear systems based on a multiple models framework has been addressed in this paper. It is based on a robust model selector that permits to identify the most representative linear model of the nonlinear system in free or faulty case. A robust isolation filter is designed to detect, isolate and estimate faults for a large class of systems through an LTI convex set representation. In order to guarantee stability of the adaptive filter, a stability analysis has been realized using linear matrix inequality formulation. The developed adaptive filter was demonstrated using a simulation example on an academic model. Moreover, the residual insensitive to fault can be used to supervise the adaptive filter itself. In the presence of model errors or uncertainties, this residual is corrupted by these perturbations and is different from zero. The adaptive filter quality can be evaluated through this residual. Futur work are directly linked to the previous remark, concerns the robustness against uncertainties and will be dedicated to industrial processes.

\section{References}

Adam-Medina, M., D. Theilliol and D. SAuter (2003). Simultaneous fault diagnosis and robust model selection in multiple linear models framework. 5 th IFAC Symposium, safeprocess, Washington D.C, USA.

Banerjee, A., Y. Arkun, R. Pearson and B. OgunNaike (1995). $h_{\infty}$ control of nonlinear processes using multiple linear models. In: $3 r d$ European Control Conference. Italy. pp. 2671-2676.

Chen, J. and R. J. Patton (1999). Robust Model-Based Fault Diagnosis For Dynamic Systems. Kluwer Academic Publishers.

DAAfouz, J. and J. Bernussou (2001). Parameter Dependent Lyapunov functions for discrete time systems with time varying parametric uncertainties. Systems and Control Letters 43, 355-359.

Frank, P. M., S. X. Ding and B. KöPPEN-SELIGer (2000). Current developments in the theory of fdi. Safeprocess'2000 IFAC Symposium, Budapest, Hungary 1, 16-27.

KELLER, J. Y. (1999). Fault isolation filter design for linear stochastic system. Automatica, 35, 1701-1706.

Leith, D. and W. Leithead (2000). Survey of gainscheduling analysis and design. International Journal of Control 73(11), 1001-1025.

MurRay-Smith, R. and T. Johansen (1997). Multiple Model Approaches to Modelling and Control. Vol. chap 8 to 12. Taylor and Francis.

NARENDRA, K., J. BALAKRishnan and M. Kermal (1995). Adaptation and learning using multiple models, switching and tuning. IEEE Control Systems Magazine, pp. 37-51.

Oliveira, M. C. De, J. Bernussou and J. C. Geromel (1999). A New Discrete-time Robust Stability Condition. Systems and Control Letters 37, 261-265.

PARK, J., G. RIZZONI and W.B. RibBENS (1994). On the representation of sensor faults in fault detection filters. Automatica 30(11), 1793-1795.

Stiwell, D. J. and W. J. RUGH (1999). Interpolation of observer state feedback controllers for gain scheduling. International Journal of Control 72(7/8), 620628.

Theilliol, D., M. Rodrigues, M. Adam-Medina and D. SAUter (2003). Adaptive filter design for fdi in nonlinear systems based on multiple model approach. 5 th IFAC Symposium, safeprocess, Washington D.C, USA.

VARGA, A. (1996). Optimal output feddback control: a multi-model approach. In: IEEE Symposium on Computer-Aided Control System Design. Dearborn, Mi. pp. 327-332. 